\title{
New Architecture for Mobile GIS Cloud Computing
}

\author{
Maged M. Elwakil ${ }^{1}$, Romani Farid Ibrahim ${ }^{2}$, Hesham A. Hefny ${ }^{3}$ \\ Computer \& Information Sciences Dept, Institute of Statistical Studies and Research, Cairo University, Giza, Egypt ${ }^{1}$ \\ High Institute of Computer Science and Information - City of Culture and Science, 6 October City, Egypt ${ }^{2}$ \\ Computer \& Information Sciences Dept, Institute of Statistical Studies and Research, Cairo University, Giza, Egypt ${ }^{3}$
}

\begin{abstract}
Nowadays the fast growth and evolution on technologies such as cloud computing, mobile computing and geographic information systems (GIS) inherently exhibit spatiotemporal phenomena. These technologies have created opportunities for business implementation that utilize advanced mapping, mobile field-based applications and location aware systems. The capability to process and handle a big volume of data using cloud computing and to access location information via mobile have made GIS technology utilizes more widely. In this paper, we introduce a new mobile GIS cloud computing architecture that increase the efficiency of processing by decreasing the transmission time for data this is based on decreasing the size of transmitted data. The mobile geographic patrolling system based on cloud GIS has great significance for the development of gas pipeline patrolling. We propose pipeline patrolling prototype system which utilizes such technology.
\end{abstract}

Index Terms: Cloud computing; mobile computing; GIS; mobile cloud computing; mobile GIS; virtual machine (VM), cloud GIS; gas pipeline patrolling.

\section{INTRODUCTION}

Mobile GIS cloud computing is one of the future technology trends. It combines the features of Mobile Computing, Geographic Information System (GIS), and Cloud Computing. The researchers predict millions of businesses will use cloud services through mobile devices by next decades, even though the recent technologies have a lack of performance. A GIS as ESRI definition is "An organized collection of computer hardware, software, geographic data, and personnel designed to efficiently capture, store, update, manipulate, analyze and display all forms of geographically referenced information". A GIS is not a standalone system, it rather provides a tool to a wide variety of application domains such as surveying and mapping, facility management, market analysis, logistics, transportation, etc. [1]. It has been utilized in representing spatial and non-spatial data [2,3]. A GIS is a data intensive system in nature and it has facilitated handling a large amount of data. The size of these data eventually will grow to an extent that these massive amount of data will hamper any organization from maintaining and handling it. Also GIS applications, which include data acquisition and preprocessing from multiple sources, are severe consumers of computational resources to perform manipulative operations and exhaustive spatial analysis [1]. Moreover, GIS is an expensive investment in hardware, software, and personnel, and data acquisition is usually costly and time consuming [4]. Therefore, in the last decades GIS is always developed through governmental authorities and massive organizations which can afford such investment. Recently many of the smaller user's different domains have the capability to drive GIS.

Nowadays mobile computing has been becoming increasingly important due to the rise of the portable

devices and the desire to have continuous connectivity to the internet irrespective of the physical location. It is a technology that enables users to access computational services anyplace, anytime, and anywhere [5, 6]. The information at a fingertip at any time and any place was only a dream. Recently this technology has become a reality using a variety of platforms, and is used by a wide range of devices such as smart phones, tablets and laptops [7]. The mobile revolution may be the biggest wave ever to hit the world of computing and it is already the biggest platform in the history of mankind [8].

We are moving into a new era of mobile computing, one that promises greater variety in applications, highly improved usability, and speedier networking such as $4 \mathrm{G}$ and LTE, which are faster and more reliable [5, 9]. In the last few years, a tremendous number of applications have been made available such as m-commerce, m-learning, $\mathrm{m}$ healthcare, m-travelling, m-GIS, etc. Recent statistical studies at the end of 2013 show that there are approximately 6.8 billion mobile subscriptions of which 1.08 billion are smart phones and there are estimated 2.7 billion people using the internet worldwide [10, 11]. A variety of sensors are built in smart phones for spatial awareness. It is now common to find a camera, a GPS module, an accelerometer, a digital compass, and a gyroscope in a single device. The GPS' ability is being tapped in Location Based Services (LBS), mapping and routing utilities, etc. Even thou philosophical implications of mobile revolution give the ability to store and retrieve mountains of information and to perform tasks on the move. Nonetheless, mobile computing still presents challenges such as devices' limited resources, networks' throughput, security standards, and power consumption 


\section{International Journal of Advanced Research in Computer and Communication Engineering}

\section{Vol. 4, Issue 10, October 2015}

that affect the deployment of such technology in sophisticated applications and manipulation of big data $[12,13]$.

Cloud computing handles such challenges. It provides resources as a service, offers scalable infrastructure and solution to host large volumes of data as well as powerful computing resources [1]. This style of computing offers dynamic scalable and virtualized resources, which provide resources, services and applications over the Internet that, are billed according to actual use [14]. Cloud computing is defined by National Institute of Standards and Technology (NIST) as a model for enabling ubiquitous, convenient and on-demand network access to a shared pool of configurable computing resources (e.g., networks, servers, storage, applications, and services) that can be rapidly provisioned and released with minimal management effort or service provider interaction [15]. The main advantages of the cloud are cost saving, high availability and elasticity. It provides almost infinite computational power and storage capacity $[14,16]$. The cloud model is composed of five essential characteristics; on-demand self-service, broad network access, resource pooling, rapid elasticity, and measured service. It is provisioned in three models; Software as a Service (SaaS), Platform as a Service (PaaS), and Infrastructure as a Service (IaaS), and it is deployed in four models; public, private, community, and hybrid clouds $[15,16]$.

The extensive use of GIS especially in mobile applications in different types of fields can be moved to the cloud with relatively a good chance $[2,3]$. The evolution of GIS from two-layer architecture to the multilayer architecture is driven by the related need of GIS applications.

The Open Geospatial Consortium (OGC) provides a blueprint for implementing GIS Service Oriented Architecture (SOA) [3, 10]. OGC facilitates the interoperability and SOA standards [10]; Web Map Services (WMS) provide implementation specification for the creation and display of map, Web Feature Services (WFS) allow retrieval, updating data and defining interfaces for data access and manipulation operations, Web Coverage Services (WCS) allow accessing part of a grid covered by a server, and the Catalogue Services for the Web (CSW) define common interface to discover, browse and query resources metadata.

The rest of this paper is organized as follows: in section IIwe present the related work. In section III, we present conceptual framework and proposed architecture. Then, we present proposed prototype model in section IV. Finally, in section $\mathrm{V}$ we provide the conclusion and future work.

\section{RELATED WORK}

The researchers Bhat et al. [3] attempt to give a description of GIS, which is widely utilized in decision support systems. In addition Cloud computing is a solution to widespread of GIS all over the world. This paper offers an assessment of GIS Cloud approach and proposes a GIS Cloud multi-tiered architecture. This architecture provides flexible solution, heterogeneous platform, scalable infrastructure, extensive business intelligent, and elastic platform to the GIS users. The study divide the GIS cloud into two major components: 1- GIS cloud web interface which is actually a web application with real time content updates. 2- GIS server that is a scalable computing resource, which is composed to communication layer, logical layer, repository layer, utilities layer and configuration layer. The paper does not present an experimental model for measuring the proposed architecture performance against GIS state of the art and the pros and cons of such experiment.

The researcher Suraj Pandey [4] presents a brief introduction of the GIS natures, which are data intensive and has a complex spatial analysis. Therefore cloud computing and its feature is required to handle such challenges. This study attempts to propose architecture comprises cloud infrastructure and GIS applications. The author presents Amazon as a cloud provider with utilizes its EC2 for computation and S3 for storage. These will distribute GIS tasks in a parallel processing and the large amount of data will use S3 API services. The Amazon VM nodes is capable to distribute user requests to a large number of worker nodes in real-time. The paper presents a comparison of traditional GIS approach and cloud GIS and its advantages such as: availability, dynamic configuration, on demand access, storage space, global access and cost. While the paper explains the benefits of using cloud, it neither illustrates its constrains and issues such as: privacy and data security, performance, service provider switching flexibility, data management, and legal aspects nor presents an experimental model to support such proposed architecture.

The researchers Aissatou Diasse and Foroski Kone [17] explain that Cloud GIS providers are facing numerous new challenges towards creating software for millions of consumers to use as a service. The architecture offers services for uploading, analyzing, editing, creating and publishing geospatial data. The main goal is to provide the power of desktop GIS solutions to a cloud GIS. This architecture provides Cloud GIS (C-GIS) anywhere in the world on demand, as a single point of access for all computing needs. The authors present a multi layers architecture contains client layer, component service layer, application service layer, software service layer and infrastructure service layer. This paper illustrate the cloud GIS advantages as easy setup, low cost, highly secure, easy publishing, modular component and interoperability. The researchers Stephen C. Hirtle and Martin [18] attempt to discuss the GIS evolution from stand-alone desktop systems to interconnected mobile devices, as well as, the GIS conversion from big purpose like urban planning, demographic analysis and topographic mapping, to small specific purpose like logistics, tourism, real estate, or marketing. The paper explains that we need to make decisions on the spot with spatiotemporal decision situations achieved by the dynamic of mobility. Location based system (LBS) is one of mobile decision support systems which help individual in dynamic and complex situations in a real-time information. Mobile decisions are supported through the initiatives of social networking and distributed information resources. This paper offers many 
to many, one to many, and many to one, information sharing to generate recommendations and decisions. The two-dimensional graphical map is the most common display on small portable devices. In addition satellite views, street-level images, and graphical mockups of the local environment are also possible. Researchers have developed tools to study the interaction between environments, individuals, and mobile devices. In addition Mobile decision-making involves a multitude of spatiotemporal constraints relating not only to people's spatiotemporal behavior in large-scale space but also to their interaction with mobile devices, and perceptual, cognitive, and social processes. The paper highlights many different ways in which the social networking is being incorporated such as volunteered geographic information (VGI), specialized Geowiki, space-time trails, place concepts as social constructs and social navigation services. In addition the research identifies the new area of maps which the invasion of image databases, allows to simply match the target location to an image. The challenge for designers will be to provide effective visual displays that increase a user's engagement with the space, which in turn leads to a greater understanding of the environment. The researchers Lingling Guo et al. [19] discuss the mobile GIS which is utilized in remote sites located in widely distribution especially in oilfields. Many field operations have the characteristic of mobility which is needed to access and control the oilfield GIS information in real time. Technology such as vector graphic has the advantages of small space usage and flexibility. Therefore, it is widely used in mobile network. Scalable vector graphics (SVG) supports image quality based on rescaling and provides the ability to edit individual elements of a graphic. Moreover, SVG has the advantage of transmitting small amounts of data to be suitable for the wireless network limited bandwidth. Oilfield GIS services create the contents that can be visualized on the mobile terminal. Mobile platform based oilfield GIS service is divided into 3 layers presentation layer, middle layer and data layer. This platform is consists of handset application and Web server program.

\section{CONCEPTUAL FRAMEWORK AND PROPOSED ARCHITECTURE WITH ARCHITECTURE LOGIC}

GIS data utilize such different approaches such as digitizer, scanner, remote sensing, satellite imagery, etc. These data has two models type vector data and raster data. Generally vector data comprise of base, thematic and infrastructure layers, while raster data as an aerial or satellite photography, which is accompanying vector data for additional ground visualization. However, these data models may be utilized in different application types or may be derived from various sources. Therefore utilization of the two models is used in different operations based on the GIS implementation.

A. Vector and Raster Data Models

The main characteristics of vector data are well represented as points, lines, or polygons, complex data structure, little storage space, topology is easily maintained, visualization is more pleasing and features are identified. Figure 1 represents an example of victor data model.

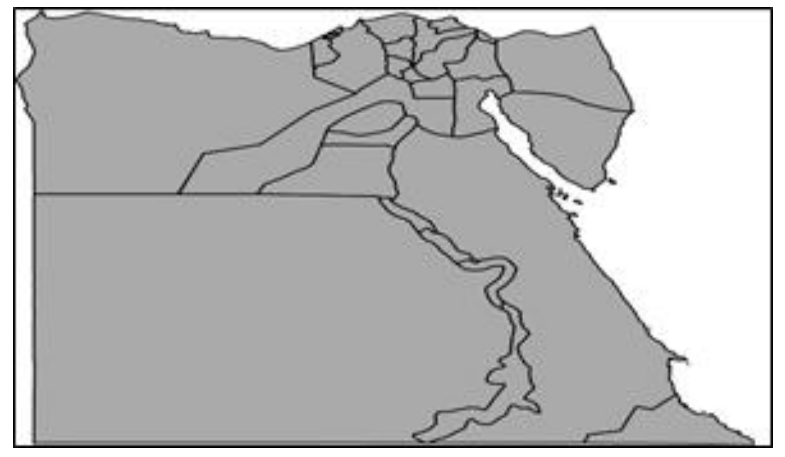

Figure 1.Example of vector data model

While raster date characteristics are simple represented as contiguous cells with different color or gray scale, simple data structure, greater storage space, topology is more difficult, visualization is not very pleasing and feature are not identified. Figure 2 represents an example of raster data model.

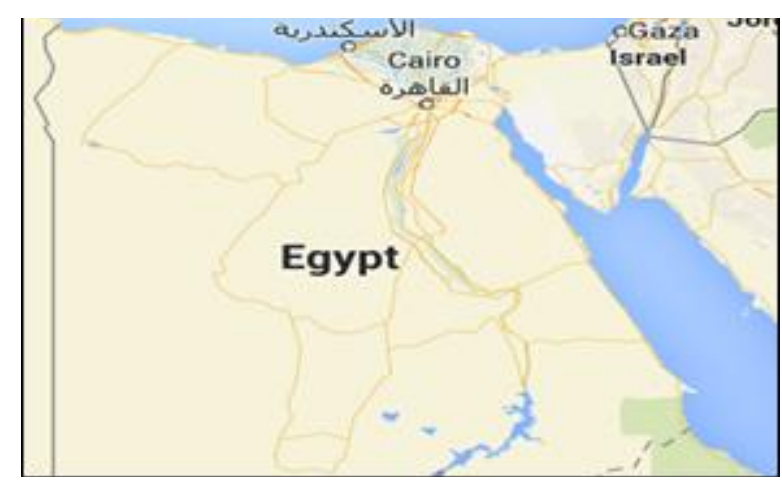

Figure 2.Example of raster data model

Technology such as Geo server is used vector model while UMN Map Server or Google map servers are utilized raster hosting. Recently, vector and raster maps are pulled together in the navigation systems. Popular navigation systems, e.g., Google Map and Baidu Map, afford raster maps to users. With the development of GIS technology, raster images are heavy size which can create transmission time, processing and storage space issues especially with mobile applications [20,21]. Therefore vector model which has less storage space and less transmission rate is more appropriate for mobility technology.

Google may overcome obtain ability and cost of suitable up-to-date cartography by providing access maps services through internet as well as real data related to roads, traffic restrictions and travel times. These free services are restricted to some limitations such as (i) The maximum size of the map tiles in pixels not exceed $1280 \times 1280$ in double scale parameter for the free API. (ii) No prefetching, caching, or content storage. (iii) No mass downloads or content bulk feeds [22, 23]. In addition these services have not taken into account constraints of data transmission rate or security issues. 
In our research we utilize vector data model for GIS functions with support of raster imaginary as Google hybrid map for visualization. The vector characteristics are more appropriate for mobile devices which have lack of resources and transmission rate should be considered.

\section{B. The Mobile GIS Cloud Architecture}

The conceptual design of the Mobile GIS Cloud architecture uses the same concept of multi-tier architecture. This architecture follows a client-server architecture in which user interface, functional process logic (business rules), GIS functionality in addition data storage and data access are developed and maintained as independent modules on separate platforms [24]. This architecture is composed in which mobile-end represents presentation, meanwhile cloud-end represents application processing, geo-processing and data access tiers. These tiers are logically separated in there processes and functions. In this architecture, clients (mobile-end) connect to the application server at cloud-end through communication tier which contains mobile service providers and internet service providers. Application server authenticates clients and performs business logic. Clients follow the logic and request spatial information from appropriate geo-servers at cloud-end. Then geoservers receive requests and perform proper GIS functions. Meanwhile database servers at separated tier in cloud-end store/retrieve or send/receive data from and to application server and geo-servers respectively. Figure 3 illustrates the proposed mobile GIS cloud architecture.

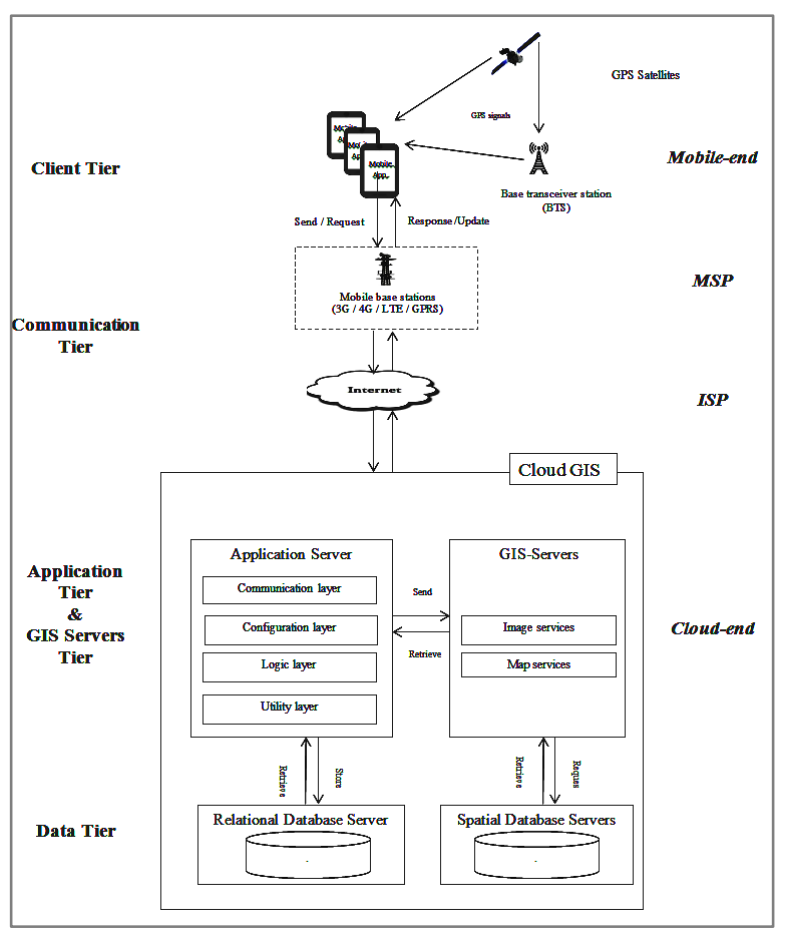

Figure 3.Mobile GIS Cloud Architecture

This architecture is designed, developed and operated as robust, scalable, and responsive architecture. The architecture is composed to five tiers:

Client tier [mobile-end]: refers to the mobile application on mobile device where users interact with spatial and nun-spatial data and analysis tools. Some of GIS functions can be accomplished based on commands, tools and tasks that are generated by client-side or server-side. In addition client tier may have some business logic associated with servers' business rules.

Communication tier: It abstracts all the communication between the handheld devices at the field (mobile-end) and application server, geospatial servers, repository at cloud-end in addition manage the communication with Global Positioning System (GPS). It utilizes technologies such as GSM, Internet, GPRS, 3G, 4G, etc. which exchange the information in real-time between mobileside and cloud-side. Meanwhile GPS is used to collect mobile location coordinates. These coordinates are transmitted to servers at cloud-end and overlay at GIS established layer at mobile-end.

Application tier: The main objective of an application tier is to separate business logic from presentation logic, GIS logic and database logic. It helps to develop, deploy and manage business rules in a distributed environment. It contains logic and relays tasks to clients. Furthermore, it acts as middleware that maintains and manages connection between mobile-end, GIS servers and databases.

GIS servers tier: It provides spatial analysis, spatial queries, geo-coding and geo-processing. An open source products such as Geoserver and Google map server are designed for interoperability and published data from any major spatial database source using open standards OGC. They deliver dynamic maps to the client based on user requests. In addition hosts GIS resources such as maps and locations coordinate. It provides such resources as services to application server and mobile-end.

Data tier: It composed of relational database server and spatial database servers. Each server manages its repository logic. Relational database server stores / retrieves data according application server processes. Meanwhile spatial database servers store geo-data and retrieve data which is requested from geoserver according to client-end or application server business logic. Product such as PostgreSQL a well-known open source can compose spatial and non-spatial data. It supports data servers such as PostGIS and ArcSDE. In addition it provisions file formats such as Shapefiles and GeoTiff, and produces output as KML, GML, SVG, Shapefiles, GeoRSS, GeoJSOn, etc.

C. Description of the architecture logic

The main objective of mobile GIS cloud architecture is to minimize the data size which is transmitted between mobile unit, application server and GIS servers. To achieve this goal, the following approach can be used as follows:

- The organization creates vector maps for all map types (paper maps and raster maps) that can be used for different applications. These vector maps are usually enough to perform the required tasks according to the business logic.

- These vector maps usually are stored on more than one GIS server (distributed GIS servers that are located in a private cloud for faster data accessing, security issues 
and no problem with handling large amount of data).

- The organization creates multilevel index for these vector maps according to the areas and the applications type (for examples Cairo, Alex, etc., and streets, regions, water pipelines, gas pipelines, etc.).

- We assume that the mobile unit is a device with limited resource capabilities such as a mobile phone, or a GUI terminal.

- The task manager assigns jobs to the mobile users, each job includes information about the location that the mobile user will go for it, vector map id for that area and the coordinates for the points where the mobile user will perform the required task.

- When the mobile user login to the system, the application server automatically checks for the assigned jobs for the mobile user and send it to the mobile unit.

- The mobile user clicks on the map id and send a request to the GIS server to retrieve the required vector map to perform the business task.

- The GIS server searches in the distributed GIS database based on the map id, and sends the requested map to the mobile unit.

- The mobile user goes to the required location according to the map with the help of the GPS which determines the path from the mobile user current location to the destination point based on the destination point coordinates.

- When the mobile user is in the correct location, he performs the required task and sends the completed checklist to the application server in the private cloud.

- In rare case, if the vector map is not enough and the mobile user need more details as a ground visualization to do his task, he can use raster map as Google maps with high quality presentation, but with the cost of long transmission time for downloading which means more processing time. Figure 4 represents this logic as an activity diagram.

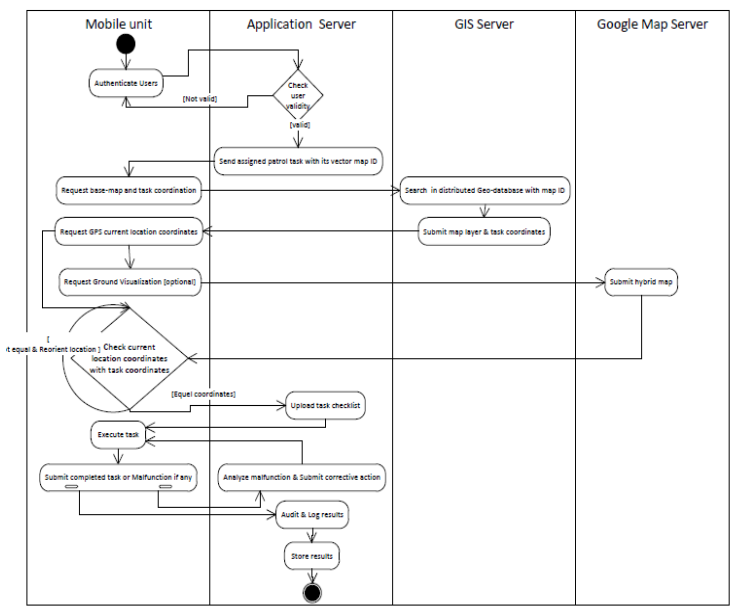

Figure4.Mobile GIS Pipeline Patrolling System Activity Diagram

\section{PROTOTYPE MODEL}

\section{A. Gas Pipeline Patrol Framework}

All regulated and standard precautions should be taken by Gas Pipeline Company to protectits pipeline and population in the neighborhood. Main objectives of pipeline patrolling are identifying the actions or activities that could damage the pipes, also identifying land slippage, third party threats, etc. in the region of the pipeline that could cause subsequent problems. In addition leakage survey is executed to protect the people and staff against gas escaping effects and detect damage to the pipeline. The frequency of the patrolling will vary for differing areas. In desert there is minimal work executed around the pipeline. In the other side, there is a lot of excavation activity in urban areas on water mains, sewers, etc. therefore the inspection frequency needs to be more rapid. The pipeline patrolling conducts two survey tasks for each day one for patrolling duties and the other for leakage survey duties. These two tasks are designed as operation needs to carry in the performance of the task. The preventive maintenance plan (PMP) should be determined in advance for both pipeline patrolling frequency and leakage survey. All reasonable precautions to reduce the risk of pipelines being struck or damaged should be taken. The inspection and surveillance applied to every particular section of a pipeline should reflect the plan and the type of frequency levels. Frequency levels should be regularly reviewed at a certain time intervals. Conduct additional patrols based on the following circumstances earthquakes, landslides, fires, heavy rainstorms, rainfalls or other disasters [25].

Real-time monitoring and management are the advantage of the mobile GIS cloud patrolling. It is improve pipeline patrol effectiveness and efficiency that guarantee safe operation and security of pipeline. As company control room can online tracing patrollers' positions and actions for safety purposes and real-time tasks accomplishment. Figure 5 illustrate the Pipeline Patrolling System Process Framework.

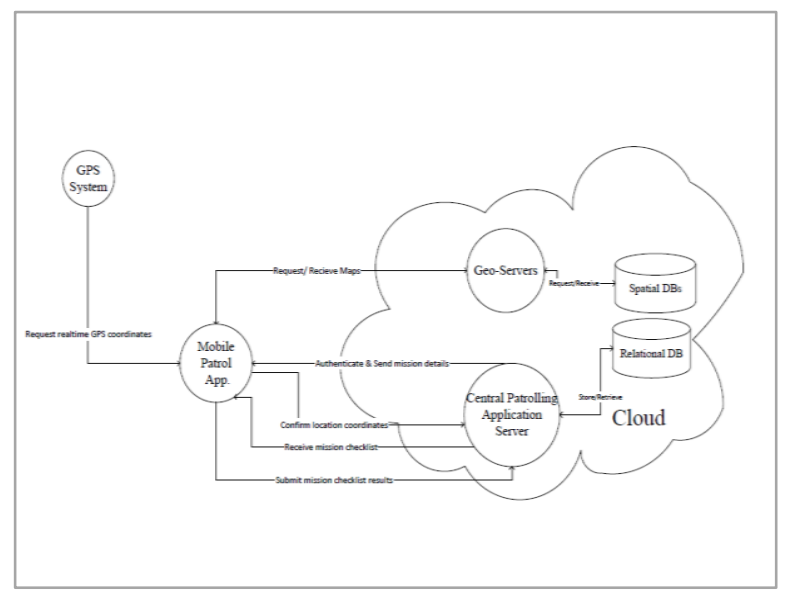

Figure 5.Pipeline Patrolling System Process Framework

\section{B. Prototype Implementation}

Mobile GIS Cloud Patrol system consists of two main components which presented activity diagram in figure 4 . 


\section{International Journal of Advanced Research in Computer and Communication Engineering}

\section{Vol. 4, Issue 10, October 2015}

1) Cloud-end contains application server, repository and GIS servers.

2) Mobile-end contains mobile application.

Pipeline Company rendering to standards prepares preventive maintenance, inspection and leakage survey patrol plan which is stored at system repository at cloudend. According to stored plane system will perform the follow:

- Application server at cloud-end Issues patrol tasks related to stored schedules.

- Application server authenticates users and allocates task to a particular patroller who will conduct these tasks.

- Related to task issued and task coordination, mobileapp will overlay a context base map based on map id through the map server at cloud-end to save transmission and processing time.

- In addition the mobile-app will render private marker on top of the tile map that is represent the task (pipeline, valve-room, cathodic protection, etc.) location.

- Mobile user can optionally switch to Google hybrid map as a raster map for ground visualization.

- Patrolman (mobile-user) at operation field obtains real time acquisition of geographical location coordinates from GPS.

- The acquired current location coordinates should matches with the stored task coordinates, whereas equivalents coordinates is a must.

- Incase coordinates does not match mobile-app will reorient and guide the user to the accurate task position by calculate the distance and the rout (if any) between user' location and task location.

- Mobile-user transmits the matched coordinates in realtime to the application server at cloud-end and requests mission details.

- Application server receives users IDs and there coordinates (double check) then transmits the patrol checkup list to users.

- Mobile-users will do the following steps:

o Fill the checklist which these data are sent to server to be logged, audited and stored in the repository.

o Send site malfunction conditions (position, time, images) if any.

- Application server will receive transmit data and process the following:

$\circ$ Patrolling and survey elicitation results for logged and audited.

o Analyze the malfunction.

$\circ$ Raise an alarm and send the corrective actions to the mobile-users.

- Finally patroller gets rid of the failure and upload condition maintenance records.

- Application server at cloud-end receives records and store in the DB.

Figures 6and 7 illustrate vector map with task and user locations mark, vector and raster map with orientation route and sample of checklist.

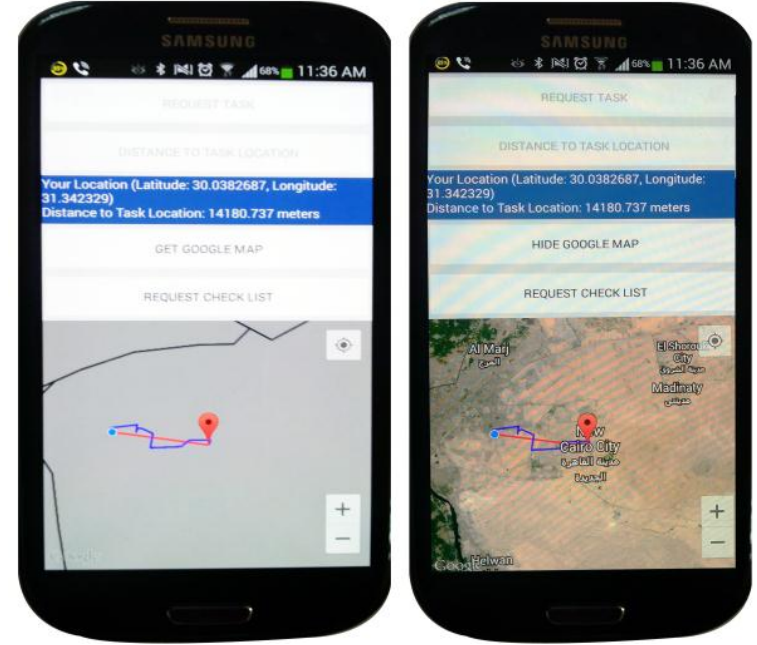

Figure 6.Vector and Raster Map with Task Location Mark \& Orientation Route

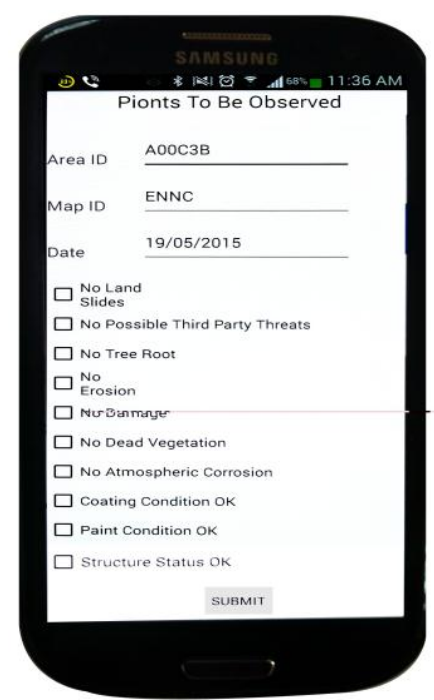

Figure 7.Sample of Checklist

\section{CONCLUSION AND FUTURE WORK}

Mobile devices have a lack of resources such as battery life time, processing and storage. These issues represent challenges to use such devices handling massive amount of data such in GIS. The mobile GIS cloud can provide a crucial role in the field-based management process through the combination of the client-side in the field (mobile components), GIS functionalities and the serverside (cloud components). However, this should be accomplished through a well-established architecture. In this paper, we presented a mobile GIS cloud architecture that will help to reduce mobile processing, storage usage and battery life, in addition utilize scalable cloud resources. This architecture is combined pool of compute and storage system to gather, manipulate, analyze, and display spatial and non-spatial data. We demonstrated that even mobile phone whose computer power is poor can interact with GIS servers and perform useful location based service. 


\title{
International Journal of Advanced Research in Computer and Communication Engineering
}

\author{
Vol. 4, Issue 10, October 2015
}

In this research, we discussed the cloud computing approach with mobile GIS applications and the benefits of implementing field-based systems. These applications improve the accessibility and usability of GIS resources. Mobile GIS prototype based on cloud computing for pipeline patrolling has been presented. This system can support field-stuff with geo-information in their mobile devices, including map browsing, query, and basic GIS functions. Furthermore the system allows field stuff to be more effective collecting critical information, increase their security and productivity. Moreover it offers online controlling across wide and span large regions. It offers a great implication in improving the development of pipeline patrolling in gas industry.

In our future research we will investigate the performance evaluation of our approach compared to other approaches. Also, management of the transaction processing between the mobile unit and the data server at the cloud computing and working offline will be investigated.

\section{REFERENCES}

[1] XiaosanG., and W. Huilian. "Cloud-based Service for Big Spatial Data Technology in Emergence Management." Proc. ISPRS, Intl Archives of Photogrammetry, Remote Sensing and Spatial Information Sciences 38, 2009.

[2] ESRI, "Understanding GIS-the ARC/INFO Method",ESRI, 1993.

[3] Muzafar Ahmad Bhat, RazeefMohd Shah, and Bashir Ahmad, "Cloud Computing: A solution to Geographical Information Systems (GIS)", International Journal on Computer Science and Engineering, P. 594-600, 2011.

[4] PandeySuraj, "Cloud Computing Technology \& GIS Applications", The Asian Symposium on Geographic Information Systems, 2010.

[5] Hoang T. Dinh,Chonho Lee, DusitNiyatoandPing Wang, "A survey of mobile cloud computing: architecture, applications, and approaches.", Wiley Online Library, 2011.

[6] Shekhar, Shashi, et al. "Spatial big-data challenges intersecting mobility and cloud computing." Proceedings of the Eleventh ACM International Workshop on Data Engineering for Wireless and Mobile Access. ACM, 2012.

[7] ParamvirBahl, Richard Y. Han, Li Erran, and Li MahadevSatyanarayanan, "Advancing the state of mobile cloud computing." Proceedings of the third ACM workshop on Mobile cloud computing and services. ACM, 2012.

[8] IgorPernek, Mostafa Abdulla Uddin, and Jack Fernando, "Hot Mobile 2012." Pervasive Computing, IEEE, October 2012.

[9] Agarwal Dinesh Agarwal, SatishPuri, Xi He, Sushil K. Prasad and Xuan Shi, "Crayons: A Cloud Based Parallel Framework for GIS Overlay Operations", Distributed \& Mobile Systems Lab, May 2011.

[10] OGC Standards and Supporting Documents, http://www.opengeospatial.org/standards.

[11] Brahima Sanou, "Measuring Information Society", International Telecommunication Union, 2013.

[12] Hung and Shih-Hao, "Executing mobile applications on the cloud: framework and issues." Computers \& Mathematics with Applications, Elsevier, 2012.

[13] Niroshinie Fernando, Seng W. Loke, and Wenny Rahayu. "Mobile cloud computing: A survey." Future Generation Computer Systems, Elsevier, 2013.

[14] Borko Furht and Armando Escalante, "Handbook of Cloud Computing", Springer Science + Business Media, 2010.

[15] Peter Mell and Timothy Grance, "The NIST definition of cloud computing", NIST special publication, 2011.

[16] Qi Zhang, Lu Cheng and RaoufBoutaba, "Cloud computing: stateof-the-art and research challenges", Journal of Internet Services and Applications, Springer, 2010, 7-18.
[17] Diasse Aissatou and Foroski Kone, "Proposed of a GIS Cloud (GIS-C) System Architecture in Private Used." Future Intelligent Information Systems. Springer Berlin Heidelberg, 2011. 55-63.

[18] Stephen C. Hirtle and Martin Raubal, "Many to Many Mobile Maps." Cognitive and Linguistic Aspects of Geographic Space. Springer, 2013.

[19] LinglingGuo, Man Yuan , and Shaobin Hu, "Oilfield GIS service based on mobile platform." Int'l J. of Communications, Network and System Sciences, April 2010, 402-405.

[20] GIS Contributor, "How Government Organizations Are Using Location Intelligence to Drive Digital Engagement", http://www.gislounge.com.

[21] V. Bhanumurthy, K. Venugopala Rao, S. Srinivasa Rao, K. Ram Mohan Rao, P. Satya Chandra, J. Vidhyasagar, P.G.Diwakar, and V.K. Dadhwal, "Enabling heterogeneous multi-scale database for emergency service functions through geoinformation technologies." ISPRS-International Archives of the Photogrammetry, Remote Sensing and Spatial Information Sciences, December 2014. 7-14.

[22] PhyoPhyoWai, Nyein Aye, and Mie MieKhin, "Lossless Compression for Raster Maps Based On Mobile" IJSRSET, Volume 1- 2015.

[23] Markus Loecher, and Karl Ropkins. "googleMaps and loa: unleashing graphics power on map tiles", Journal of Statistical Software,January 2015.

[24] SnehaRao and Sri Vinay. "Choosing the right GIS framework for an informed Enterprise Web GIS Solution" 13 Annual International Conference and exhibition on Geospatial Information Technology and Applications", Gurgaon, India; January, 2010, 19 - 21.

[25] Petroleum Safety \& Environmental Services Company, "Environmental Impact Assessment Study for El Tina- Abu Sultan Onshore Gas Pipeline Project" Egyptian Natural Gas Co. (GASCO), October 2005.

\section{BIOGRAPHIES}

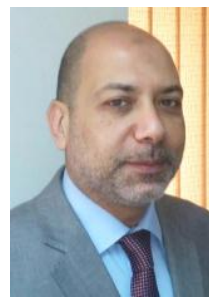

Maged M. Elwakil received Diploma and Pre-Master Degrees in computer science and information system from the Institute of Statistical Studies \& Research - Cairo University. He is a Master student affiliated with the Department of Computer Science\& Information System of Institute of Statistical Studies \& Research - Cairo University. His main research interests are mobile computing, cloud computing and GIS.

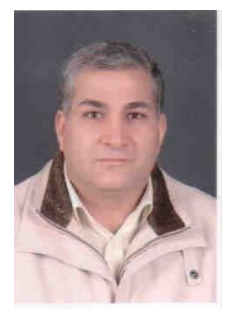

Romani Ibrahim received the Ph.D. degree in information systems from Cairo University, Egypt. He works in the High Institute of Computer Science and Information -City of Culture and Science- 6 October City, and as a part time lecturer in the Institute of Statistical Studies \& Research - Cairo University. He is a member of ACM, his research interests include distributed and mobile database systems, transaction processing, data warehousing, Cloud Computing, and information security. 